\title{
Comparing stimulation requirements and final outcome between early follicular and mid luteal pituitary suppression in the long gonadotropin releasing hormone agonist protocol
}

\author{
Abdulmagid Sarhan ${ }^{1}$, Mervat Harira ${ }^{1}$, Sherine Elshazly ${ }^{1}$, Ahmad Nouh² \\ ${ }^{1}$ Zagazig University, Egypt \\ 2Zagazig University Student Hospital, Egypt
}

\begin{abstract}
Objective: To compare stimulation requirements and ICSI outcome when agonist treatment is started in the early follicular phase or in mid luteal phase of the cycle.

Methods: 181 infertile patients were randomly assigned to: group $A(N=66)$ and group $B(N=115)$. GnRH-a (Triptorelin) subcutaneous daily injections started on day 20-22 of the previous cycle till pituitary suppression is achieved where gonadotropins stimulation commenced. In group $A$, agonist treatment was started on the first or second days of the cycle, in group B it was started on day 20-22 of the cycle. The agonist treatment was continued till the day of (hCG) administration.
\end{abstract}

Results: The stimulation requirements were similar in the two groups. The days of $t$ agonist treatment required to reach pituitary suppression were higher in group $A$ : $12.5 \pm 6.4$ than in group $B, 11 \pm 4.5$. Days of stimulation (10.4 \pm 1.7 and $10.3 \pm 1.6)$ and number of gonadotropin vials (40.1 \pm 8.7 and $39.3 \pm 9.5)$ did not differ between both groups.

The mean number of oocytes retrieved, mean number of embryos produced (11.7 \pm 7.4 and $13.3 \pm 9.3)(5.9 \pm 4.2$ and $6 \pm 5.2)$ were similar in both groups. The rates of fertilization and cleavage were similar in the two groups. Pregnancy rates were similar in both groups. The clinical pregnancy rates per cycle was $31.8 \%$ and $33 \%$, while pregnancy rates per embryo transfer was $36.2 \%$ and $36.5 \%$ in groups $A$ and $\mathrm{B}$ respectively.

Conclusion: Starting pituitary suppression with GnRH agonist in the early follicular phase or mid luteal phase were comparable regarding stimulation requirements and final outcomes.

Keywords: GnRH agonist protocol, early follicular, mid luteal, pituitary suppression

\section{INTRODUCTION}

Controlled ovarian stimulation for assisted reproduction utilizes gonadotropin releasing hormone agonist ( $\mathrm{GnRH}-\mathrm{a}$ ) routinely, most commonly in its long protocol. It was demonstrated by many randomized clinical trials that in IVF-ET, the combination of exogenous gonadotropin plus ( $\mathrm{GnRH}-\mathrm{a})$, for the suppression of pituitary gonadotropins secretion, is associated with higher pregnancy rates when compared to the use of gonadotropins without GnRH-a. Among the important benefits of these drugs are decreased cancellation rates through the prevention of premature LH surge and luteinisation (Caspi et al., 1989), recovery of a larger number of oocytes, through enhancement of follicular recruitment (Liu et al., 1992; Daya, 2000), and the improvement in routine patient treatment schedule (Zorn et al., 1987).

Pituitary suppression with the agonists is usually started in the luteal phase of the cycle. The gold standard for ovarian stimulation in young normo-gonadotropic women is recognized as the long protocol, starting $\mathrm{GnRH}-\mathrm{a}$ in the mid luteal phase of the preceding cycle day $20-22$ to achieve ovarian activity suppression before gonadotropin is administered (Maheshwari et al., 2011).

The objective of this prospective randomized controlled study was to compare the stimulation requirements and the outcome of the intracytoplasmic sperm injection (ICSI) when treatment with the agonist is started in the early follicular phase as compared to the mid luteal phase of the cycle.

\section{MATERIALS AND METHODS}

181 infertile patients were randomly assigned to two groups using a computerized randomization system through odd and even numbers; group $A(N=66)$ and group $B(N=115)$. In group $A$, treatment with the agonist was started on the first or second days of the menstrual period where patients in group $B$ started treatment on day 20-22 of the cycle. In both groups, the agonist treatment in the form of subcutaneous daily injections of $\mathrm{GnRH}-\mathrm{a}$ (Triptorelin, 0.1mg Decapepty, Ferring, Sweden) was continued till pituitary suppression was achieved, when stimulation with gonadotropins were commenced and continued till the day of human chorionic gonadotropins (hCG) administration.

Stimulation was performed using human menopausal gonadotropin (Merional, IBSA, Italy) or purified urinary FSH (Fostimon, IBSA, Italy) starting with a daily dose of 2 vials, and adjusted according to ovarian response. Ovarian response was monitored frequently by transvaginal ultrasonography. When there were two or more follicles with a mean diameter of $>17 \mathrm{~mm}$ and serum estradiol (E2) level of $\geq 200 \mathrm{Pg} / \mathrm{ml}$ per mature follicle, 10,000 IU of hCG (Choriomon, IBSA, Italy) was administered.

Transvaginal oocyte retrieval was performed $35 \mathrm{~h}$ after hCG injection. Intra-cytoplasmic Sperm injection (ICSI) was performed on metaphase II oocytes according to the conventional protocol. Embryo transfer was performed vaginally under abdominal ultrasound guidance on the second or third day after retrieval. Up to four embryos were transferred. The luteal phase support was started on the day of embryo transfer by either vaginal progesterone $400 \mathrm{mg}$ (Cyclogest, Actavis Barnstaple EX32, UK) o intramuscular progesterone $100 \mathrm{mg}$ (Prontogest, EIPCO, Egypt) according to patient preference.

Clinical pregnancy was defined by the presence of intrauterine gestational $\operatorname{sac}(\mathrm{s})$ with pulsating heart beats on transvaginal ultrasound scan at 5-6 weeks' gestation with increasing serum B-hCG levels.

Serum levels of $\mathrm{LH}$ and E2 were measured daily with commercially available enzyme immunoassay kits (Cobas, Elecsys, Enzymun, Roche diagnostics GmbH, Sandhofer Strasse116, Germany). 
The demographic data of the patients, hormonal levels, stimulation requirements and outcome variables were compared for the three groups. Results are presented as mean \pm standard deviation. Where appropriate, $x 2$ test and t-test were used for analysis using SPSS for Windows version 11.0 (SPSS Inc., Chicago, IL USA). $P<.05$ was considered statistically significant.

\section{RESULTS}

The mean age of the patients and the mean duration of infertility did not differ between the two groups. The distribution of the different causes of infertility was similar in the two groups. The basal levels of LH and E2 were similar in the 2 groups (table 1 ).

The stimulation requirements were similar in the two groups. The days of treatment by the agonist required to reach pituitary suppression were higher in group $A$, $12.5 \pm 6.4$ than in group $B, 11 \pm 4.5$ but the difference was not significant. Days of stimulation (10.4 \pm 1.7 and $10.3 \pm 1.6)$ and number of vials of gonadotropins used $(40.1 \pm 8.7$ and $39.3 \pm 9.5)$ did not differ between the two groups (table 2 ).

The mean number of oocytes retrieved $(11.7 \pm 7.4$ and $13.3 \pm 9.3)$ and the mean number of embryos produced $(5.9 \pm 4.2$ and $6 \pm 5.2)$ were similar in the two groups. The rates of fertilization and cleavage were similar in the two groups. Pregnancy was achieved in similar percentage of the two groups. Clinical pregnancy rate per initiated cycle was $31.8 \%$ and $33 \%$ while pregnancy rate per embryo transfer was $36.2 \%$ and $36.5 \%$ in groups $A$ and $B$ respectively (table 3 ).

\section{DISCUSSION}

Controlled ovarian stimulation for assisted reproduction utilizes gonadotropin releasing hormone agonist ( $\mathrm{GnRH}-\mathrm{a}$ ) routinely either in its short (flare-up) or long protocol.

The prevention of spontaneous LH surge leading to cycle cancellation is the main benefit obtained by the use of the GnRH agonist (Broekmans et al., 1996). Such a benefit explained the marked improvement of IVF results associated with the introduction of its use. As shown by placebo-controlled studies on GnRH agonists, LH surges occur in $20 \%$ of IVF patients, leading to cancellation of the IVF cycles (Edwards et al., 1996; Janssens et al., 2000). There are three main protocols involving the administration of GnRH: short, ultrashort and long protocols. The short and ultrashort protocols involve the administration of GnRHa from day 1 or day 2 of the cycle for 3 days in the ultrashort protocol (hence using only the flare-up effect) and until human chorionic gonadotrophin (hCG) injection in the short protocol. The long protocol involves the administration of GnRHa for at least 14 days to achieve ovarian activity suppression before gonadotropin is administered (Maheshwari et al., 2011).

There are two long protocol regimens; the long luteal phase protocol starts GnRHa from the mid-luteal phase of the previous menstrual cycle, whereas the long follicular phase protocol starts GnRHa from the first day of the menstrual cycle. Mahishwari et al. (2011) performed a systematic meta analysis for seven studies that showed a significant increase in the number of oocytes retrieved of up to $60 \%$, with no significant difference regarding pregnancy rate when comparing the long protocol versus the short and ultra short protocols, making the long protocol

Table 1. Demographic data from the study groups

\begin{tabular}{|l|c|c|c|}
\hline \multicolumn{1}{|c|}{$\begin{array}{c}\text { Group } \\
\text { Demographic Data }\end{array}$} & Group A (N=66) & Group B (N=115) & Value \\
\hline Age (years) & $34.6 \pm 3.2$, & $34.6 \pm 3.8$ & $\mathrm{NS}$ \\
\hline Duration of infertility (years) & $7.4 \pm 0.8$, & $3.6 \pm 0.6$ & $\mathrm{NS}$ \\
\hline Basal LH $(\mathbf{m I U} / \mathbf{m l})$ & $3.8 \pm 0.4$ & $3.6 \pm 0.3$ & $\mathrm{NS}$ \\
\hline Basal E2 $(\mathbf{P g} / \mathbf{m l})$ & $49 \pm 3.1$ & $49 \pm 3$ & $\mathrm{NS}$ \\
\hline
\end{tabular}

Table 2. Stimulation requirements of study groups

\begin{tabular}{|l|c|c|c|}
\hline \multicolumn{1}{|c|}{ Group } & Group A (N=66) & Group B (N=115) & $P$ Value \\
\hline Days to stimulation & $12.5 \pm 6.4$ & $11 \pm 4.5$ & $\mathrm{NS}$ \\
\hline Days of stimulation & $10.4 \pm 1.7$ & $10.3 \pm 1.6$ & $\mathrm{NS}$ \\
\hline Number of vials & $40.1 \pm 8.7$ & $39.3 \pm 9.5$ & $\mathrm{NS}$ \\
\hline
\end{tabular}

Table 3. Final outcome of study groups

\begin{tabular}{|l|c|c|c|}
\hline \multicolumn{1}{|c|}{ Group } & Group A (N=66) & Group B (N=115) & $P$ Value \\
\hline Cancellation & $1(1.5 \%)$ & NS \\
\hline Retrieval cycles & $65(98.5 \%)$ & $113(98.3 \%)$ & $\mathrm{NS}$ \\
\hline ET Cycles & $58(87.8 \%)$ & $104(90.4 \%)$ & $\mathrm{NS}$ \\
\hline Oocytes & $11.7 \pm 7.4$ & $13.3 \pm 9.3$ & $\mathrm{NS}$ \\
\hline Embryos & $5.9 \pm 4.2$ & $6 \pm 5.2$ & $\mathrm{NS}$ \\
\hline Pregnancy & 21 & $33 \%$ & $\mathrm{NS}$ \\
\hline Pregnancy Per cycle (\%) & $31.8 \%$ & $36.5 \%$ & $\mathrm{NS}$ \\
\hline Pregnancy Per ET (\%) & $36.2 \%$ & & 2 \\
\hline
\end{tabular}


preferable for normal responder patients undergoing IVF. Sometimes, the time factor for patient and availability of the couple for treatment may be a problem.

The objective of this prospective randomized controlled study was to compare the stimulation requirements and the outcome of the intracytoplasmic sperm injection (ICSI) when treatment with the agonist is started in the early follicular phase as compared to the mid luteal phase of the cycle. We reached that, there was no significant difference regarding pregnancy rate, number of gonadotropin vials, number of oocytes and days of stimulation.

The same conclusion was supported by a systematic meta-analysis done by Maheshwari et al. (2011) where there was no significant difference in pregnancy rates in follicular start of GnRHa use when compared to luteal start. In a study done by Urbancsekon \& Witthaus (1996), no significant differences was found between day 1 and day 21 onset as regarding to ovarian response, cancellation, fertilization, and pregnancy rates. The authors concluded that both follicular and luteal phase initiation of GnRH-a long-protocol down-regulation are equally efficacious, but starting on day 1 is more easily recognizable by patients and avoids the possibility of administering $\mathrm{GnRH}-\mathrm{a}$ in the presence of an unsuspected pregnancy.

Pellicer et al. (1989) found that there was no difference between the two protocols regarding the dose of gonadotropins necessary to reach an optimal response, fertilization and cleavage rates. Three other studies performed by Pellicer et al. (1989), Ron-El et al. (1990) and Kondaveeti-Gordon et al. (1996) reported that there was evidence of higher amounts of gonadotropins required in the luteal start (Janssens et al., 2000)

In conclusion, the two timing of starting the agonist were sufficiently potent to produce pituitary suppression in a short period of time. Stimulation requirements as the number of gonadotropin vials and the days of stimulation required to reach the criteria of hCG administration were comparable in the two groups. Other factors should be considered, such as the couple's availability and the workload of the center.

\section{CONFLICT OF INTERESTS}

No conflict of interest have been declared.

\section{Corresponding author:}

Abdulmagid Sarhan

Professor, Zagazig University, Egypt

E-mail: Sarhan_a@hotmail.com

\section{REFERENCES}

Broekmans FJ, Hompes PGA, Lambalk CB, Schoute E, Broeders A, Schoemaker J. Short term pituitary desensitization: effects of different doses of the gonadotrophin-releasing hormone agonist triptorelin. Hum Reprod 1996;11: 55-60.

Caspi E, Ron-El R, Golan A, Nachum H, Herman A, Soffer $Y$, Weinraub $Z$. Results of in vitro fertilization and embryo transfer by combined long-acting gonadotropin-releasing hormone analog D-Trp-6-luteinizing hormone-releasing hormone and gonadotropins. Fertil Steril. 1989; 51: 95-9.

Daya S. Gonadotropin releasing hormone agonist protocols for pituitary desensitization in in vitro fertilization and gamete intrafallopian transfer cycles. Cochrane Database Syst Rev 2000; 2: CD001299.

Edwards RG, Lobo R, Bouchard P. Time to revolutionize ovarian stimulation. Hum Reprod 1996;11:917-19.

Janssens RMJ, Lambalk CB, Vermeiden JPW, Schats R, Bernards JM, Rekers-Mombarg LT, Schoemaker J. Dose finding study of triptorelin acetate for prevention of a premature LH surge in IVF: a prospective, randomized, double-blind, placebo-controlled study. Hum Reprod 2000; 15: 2333-40.

Kondaveeti-Gordon U, Gordon CA, Harrison RF, Drudy L, Barry-Kinsella C, Cottell E. A randomized prospective study of early follicular or mid luteal initiation of long protocol gonadotropin-releasing hormone in an in vitro fertilization program. Fertil Steril.1996; 66:582-86

Liu HC, Lai YM, Davis O, Berkeley AS, Graf M, Grifo J, Cohen J, Rosenwaks Z. Improved pregnancy outcome with gonadotropin releasing hormone agonist ( $\mathrm{GnRH}-\mathrm{a})$ stimulation is due to the improvement in oocyte quantity rather than quality. J Assist Reprod Genet. 1992; 9: 338-44.

Maheshwari A, Gibreel A, Siristatidis CS, Bhattacharya S. Gonadotrophin-releasing hormone agonist protocols for pituitary suppression in assisted reproduction. Cochrane Database Syst Rev. 2011; 8:CD006919.

Pellicer A, Simon C, Miro F, Castellvi RM, Ruiz M, Perez $M$, Bonilla-Musoles $F$. Ovarian response and outcome of in vitro fertilization in patients treated with gonadotrophin-releasing hormone analogues in different phases of menstrual cycle. Human Reproduction 1989;4: 285-9.

Ron-El R, van der Ven H, Herman A, Caspi E, Golan A, Diedrich $\mathrm{K}$. The comparison of early follicular and mid luteal administration of long-acting gonadotrophin-releasing hormone agonist. Fertil Steril. 1990; 54:233-37.

Urbancsekon J, Witthaus E. Midluteal buserelin is superior to early follicular phase buserelin in combined gonadotrophin releasing hormone analogue and gonadotropin stimulation in in vitro fertilization. Fertility and Sterility 1996;65: 966-71.

Zorn JR, Boyer P, Guichard A. Never on a Sunday programming for IVF-ET and GIFT. Lancet. 1987; 329:385-86. 